\title{
Movimiento y regímenes de vitalidad. La nueva organización de la vida en la biomedicina
}

\author{
Movement an regimes of vitality. New organization of life in medicine
}

\author{
Francisco TIRADO \\ Universitat Autónoma de Barcelona \\ franciscojavier.tirado@uab.es \\ Ana GÁlvez \\ Universitat Oberta de Catalunya \\ agalvez@uoc.edu \\ Jorge CASTILLO ${ }^{1}$ \\ Universitat Autónoma de Barcelona \\ jcastillo.s@hotmail.com
}

Recibido: 16.02 .2012

Aprobado definitivamente: 02.10 .2012

\section{RESUMEN}

El texto plantea que la masiva protocolarización de la biomedicina ha creado una nueva materialidad para la patología. Ésta es caracterizada a partir de la noción de "objeto potencial" que acuñó A.N.Whitehead. Los objetos potenciales constituyen una completa y heterogénea ordenación de la vida. A tales órdenes resultantes de la aparición de un objeto potencial se les denomina "regímenes de vitalidad". Éstos se caracterizan por establecer las diferentes escalas de la vida en la actividad médica, la temporalidad de los procesos médicos, por establecer el tipo de relación entre normalidad y anormalidad y, finalmente, por generar un conjunto de condiciones de motilidad para los individuos. A partir de una serie de investigaciones sobre los protocolos médicos del cáncer de mama, los que rigen emergencias sanitarias como las epidemias o implementan la teleasistencia sostendremos que el movimiento es indisociable del juego de verdad que ofrecen los regímenes de vitalidad. También argüiremos que la biomedicina, al hacer lo anterior, ha sustraído la preocupación por el movimiento del plano individual e incluso político y lo ha vinculado con el de la salud, lo científicamente prescriptible y normativo. En suma, ha transformado la movilidad en buen-y-sano-movimiento.

Palabras clave: Movilidad, biomedicina, régimen de vitalidad, objeto potencial, organización de la vida

\begin{abstract}
This paper puts forward that the massive use of protocols in biomedicine has created a new kind of materiality for pathology. This is very well depicted by the notion of 'potential object' coined by A.N.Whitehead.

\footnotetext{
${ }^{1}$ Programa de Doctorado en Psicología Social, Departament de Psicología Social, Universitat Autònoma de Barcelona
} 
Potential objects are a complete and heterogeneous ordering of life. We will call to these orderings, resulting from the shaping of such potential objects, regimes of vitality. They establish different scales of life, actors and the relation between them in biomedical activity. Moreover, they define the temporality in the medical processes, the correct articulation between normality and abnormality and, finally, they produce a set of motility conditions for individuals. Based on a research about protocols on cancer, telecare and epidemics we will pose that movement is inseparable from the "truth" that always appears in the regimes of vitality. In this sense, we will also pose that biomedicine has remove our concern about movement from the political and individual sphere and has put it in the sphere of health and normativity of science. In a nutshell, biomedicine has transformed mobility into correct-and-healthy-movement.

KEYwORDs: Mobility, biomedicine, regime of vitality, potential object, organization of life

\section{SUMARIO}

1.La protocolarización de la medicina. 2.Objetos potenciales y regímenes de vitalidad. 3.Regímentes de vitalidad y motilidad. 4.Conclusiones: una nueva organización de la vida 
Nos gustaría abrir estas páginas con tres noticias de prensa relativamente recientes y un interrogante. Las noticias son:

\section{El gobierno introduce regulaciones estrictas} de control.

Lahora [Pakistán]: El Departamento de Salud presentó el Jueves el Reglamento de Control de Dengue 2011 en virtud de la Ley de Control Epidémico de Punjab de 1958, para entregar protección legal a las medidas que ha adoptado para combatir la enfermedad transmitida por mosquitos... las regulaciones harían obligatorio seguir en las escuelas las directrices gubernamentales sobre el control del dengue. Las normas se mantendrían en rigor hasta el 30 de Noviembre de 2011, tiempo durante el cual se elaborará cualquier legislación necesaria [...] las regulaciones requieren que los dueños de comercio de neumáticos, talleres, viveros y sitios de construcción, "destruyan todos los elementos que pueden ayudar a la expansión del dengue". Los alcantarillado serían desatascados y los estanques artificiales secados una vez por semana para prevenir la proliferación de criaderos de mosquitos. (Gov introduces stringent control regulations, 2011, traducción de los autores)

\section{Cruz Roja probará en Sarria un nuevo sistema de teleasistencia.}

La asamblea local de la Cruz Roja de Sarria probará un nuevo sistema de teleasistencia, de segunda generación, que cuenta con un detector de incendios y movimiento... Los participantes en este plan piloto tendrán un botón de alarma como en otros sistemas de este tipo que pueden pulsar si sufren algún problema... Si bien, el nuevo sistema cuenta con un sensor que avisa en caso de que se produzca un incendio en la vivienda. También tiene un detector de movimiento y si la persona se levanta de la cama por la noche y tarda en regresar a su dormitorio saltará la alarma. El sistema se pone en contacto con una operadora, ubicada en A Coruña, que se comunica con la vivienda para saber si se produjo algún tipo de incidente. (Cruz Roja probará en Sarria un nuevo sistema de teleasistencia, 2011)

\section{Estar sentados durante mucho tiempo puede aumentar el riesgo de cáncer.}

Pasar mucho tiempo sentados aumenta el riesgo de morir prematuramente y de padecer cáncer, incluso en el caso de quienes se ejercitan diariamente. El dato sale de un estudio realizado a 123 mil estadounidenses y fue dado a conocer en la última conferencia... que analizó el efecto del sedentarismo en esta enfermedad... La relación directa entre actividad física y cáncer estaría dada por la acumulación de grasa provocada por la falta de movimiento, lo que tendría un efecto en el desarrollo de ciertos tipos de cáncer, como el de colon, el de mama y el de endometrio. (Nanjari, 2011)

Y el interrogante: ¿qué relaciona a estas piezas de información más allá de su actualidad? La respuesta evidente es que las tres hacen referencia a situaciones sanitarias o eventos de salud. ¡Es correcto! No obstante, comparten mucho más. En primer lugar apuntan hacia una transformación radical en la práctica médica. En segundo, señalan cómo tal transformación conlleva, a su vez, un cambio de dimensiones insospechadas en nuestra vida cotidiana más inmediata. En tercer lugar, las tres hablan del despliegue de una nueva organización de la vida.

Efectivamente, en las últimas dos décadas diversos estudios han documentado profundos e impactantes cambios en la práctica médica. Éstos tienen que ver con desplazamientos epistemológicos, con modificaciones en sus formas de organizar la actividad médica y sus métodos y con la emergencia de nuevos enfoques sobre su praxis (Rose, 2007; Keating \& Cambrosio, 2003, 2000; Rheinberger, 2000). Entre toda esta literatura destaca la que hace referencia a la mutación de la medicina en tanto que conocimiento científico. Semejante transformación se expresaría a partir de cuatro dimensiones. La primera hace referencia a la conversión de la medicina en biomedicina (Bourret, Keating, \& Cambrosio, 2011; Keating \& Cambrosio, 2003). Ésta organiza su producción de conocimiento a partir de unos macro-complejos sociales y epistémicos denominados "plataformas biomédicas" (Keating \& Cambrosio, 2000 ), produce y recurre a un nuevo tipo de objetivi- 
dad denominada "objetividad regulatoria" (Moreira, May \& Bond, 2009; Cambrosio, Keating, Sclhlich $\&$ Weisz, 2006) y despliega una severa mirada molecular sobre el fenómeno de la vida misma (Rose, 2007, 2001). Si durante siglos la medicina se ocupó de lo anormal y lo patológico, en este momento, la biomedicina despliega sus saberes y tecnologías en el terreno de la modificación y mejora de las capacidades normales del individuo. En ese sentido, podemos afirmar que la segunda dimensión tiene que ver con el hecho de que la biomedicina no opera ni tratando la patología ni previniéndola. Su actividad se centra en un tipo de acción preparatoria ${ }^{2}$ que busca definir cualquier posibilidad futura y reproducirla en el presente para evitar que alguno de esos futuros se torne realidad (Rose, 2007). Es decir, la biomedicina es una actividad médica pre-sintomática, opera antes de que aparezcan los síntomas y señales de la patología. La tercera dimensión muestra que ha emergido recientemente un nuevo papel para las asociaciones de pacientes y para la propia figura del paciente. Diversos autores han denominado a este fenómeno "política de la vitalidad" (Landzelius, 2006). En este ejercicio político el paciente es un actor completamente activo que desea tener voz e implicarse en la propia producción de un conocimiento y unas tecnologías médicas que en última instancia recaerán sobre su cuerpo y su vida cotidiana (Callon \& Rabeharisoa, 2008).

Las anteriores transformaciones constituyen el marco de la investigación que presentamos en este artículo. ${ }^{3}$ Plantearemos que es posible descri- bir transformaciones en la medicina que se suman a las descritas anteriormente si se realiza un detallado análisis de la materialidad de la biomedicina. Sin embargo, aunque pueda parecer paradójico, cuando hacemos referencia a tal materialidad no tenemos presente el conjunto de artefactos técnicos, objetos de laboratorio, instrumental quirúrgico o espacios que siempre han acompañado a la praxis médica. Nos referimos, por el contrario, a algo tan simple y aparentemente modesto como un protocolo o guía de actuación. Si alguna característica general distingue a la biomedicina y no ha sido suficientemente enfatizada en los estudios mencionados anteriormente es su absoluta dependencia del protocolo o guía. Como mostraremos en el primer apartado de este texto, el fenómeno de la protocolarización es masivo y caracteriza la actividad médica de los últimos veinte años. En el segundo epígrafe argüiremos que un protocolo o guía es mucho más que unas cuantas hojas de papel o un conjunto de instrucciones procedimentales. Concretamente, es una acción que convierte el fenómeno patológico en un "objeto potencial" y ofrece a la enfermedad una nueva materialidad. Tal concepto fue acuñado por Alfred North Whitehead (1925) y hace referencia a objetos que operan como lo haría un campo magnético o gravitacional. Es decir, el objeto está en todas partes al mismo tiempo, actuando con una eficiencia positiva y real simultánea. No es un objeto virtual o una potencia que se actualiza a partir de determinadas operaciones. El objeto potencial siempre se define por su presencia y actualidad. Plantearemos que el trabajo de la biomedicina

\footnotetext{
${ }^{2}$ Los autores mencionados señalan que la medicina actual ha entrado en una lógica caracterizada por una "preparedness" (preparatividad) que la lanza a una acción sobre el futuro antes que sobre las patologías presentes.

${ }^{3}$ Los resultados que exponemos en este texto provienen de tres investigaciones. La primera es el estudio de caso de una asociación creada por un grupo mujeres afectadas de cáncer de mama en la ciudad de Barcelona, gAmis (Grup d'Ajuda Mama i Salut). El estudio ha contemplado la realización de etnografías focales (Knoblauch, 2005) en el curso de 18 meses, recopilando y analizando además una serie de protocolos y llevando a cabo entrevistas en profundidad tanto a las asociadas como a profesionales del ámbito oncológico y de rehabilitación. La segunda es un estudio de caso de la situación de emergencia que originó la epidemia de gripe H1N1. En éste se analizaron los documentos oficiales sobre la misma, los protocolos de actuación médica y la información aparecida en diversos medios de comunicación nacionales e internacionales. La tercera es un trabajo sobre la implementación de servicios de teleasistencia y telecuidado en Barcelona. En él se llevaron a cabo etnografías y entrevistas con profesionales y usuarios. En esta última participaron otros investigadores de la Universitat Autònoma de Barcelona y la Universitat Oberta de Catalunya (Miquel Domènech, Daniel López, Blanca Callén, Tomás Sánchez Criado y Nissaia Cassén) y tuvo financiación estatal (Ministerio de Educación y Ciencia: CSO2008-06308-C02-01/SOCI) e internacional (Unión Europea: Project Number 217787 / FP7-SCIENCE-IN-SOCIETY-2007-1). A lo largo de estas páginas aparecen ejemplos extraídos de las anteriores entrevistas o de los protocolos y guías analizados.
} 
se traduce en la producción constante de semejantes objetos. Ellos son relevantes porque constituyen una completa y heterogénea ordenación de la vida. Y denominaremos a tales órdenes "regímenes de vitalidad". Éstos se caracterizan por establecer las diferentes escalas de la vida en la actividad médica, la temporalidad de los procesos médicos, por establecer el tipo de relación entre normalidad y anormalidad y, finalmente, por generar un conjunto de condiciones de motilidad para los individuos. En el tercer apartado del texto analizaremos el fenómeno de la movilidad como algo inscrito y dependiente de un régimen de vitalidad. Desde este punto de vista sostendremos que el control biopolítico de la movilidad pasa, entre otras cosas, por establecer el mencionado conjunto de condiciones de motilidad como una verdad vinculada a su correspondiente régimen de vitalidad. Aclararemos que diferenciamos entre motilidad y movilidad para distinguir entre las condiciones de posibilidad del movimiento y su realización efectiva. También argüiremos que la biomedicina, al hacer lo anterior, ha sustraído la preocupación por el movimiento del plano individual e incluso político y lo ha vinculado con el de la salud, lo científicamente prescriptivo y normativo. $\mathrm{Y}$ en esa vinculación ha convertido la movilidad en una dimensión en la que la motilidad y lo económico se superponen. El movimiento deja de ser una mera cualidad de los seres biológicos para convertirse en algo que se puede generar, potenciar, combinar, ofrecer, vender... Es decir, se convierte en cinevalor.

\section{LA PROTOCOLARIZACIÓN DE LA MEDICINA}

Una de las revoluciones más importantes que ha sufrido la medicina en las últimas décadas es la aparición de la denominada "Medicina Basada en la Evidencia" (Evidence-Based Medicine) (Knaapen, Cazeneuve, Cambrosio, Castel, \& Fervers, 2010; Timmermans, \& Kolker, 2004). Este movimiento vindica esencialmente un fortalecimiento científico de los fundamentos de las actividades de cuidado y las prácticas clínicas. Su punto de partida es el diagnóstico de una situación de debilidad de la práctica médica caracterizada por tres graves problemas. En primer lugar, multitud de estudios clínicos muestran la continua diferenciación de criterio y aplicación en las prácticas clínicas, evento que conlleva una pérdida de credibilidad de las mismas. En segundo, la calidad del cuidado y la clínica parecen resentirse de la anterior proliferación permanente de patrones de atención. Por último, se acumulan gastos redundantes y se pierde el control del coste de tales prácticas (Sackett, Rosenberg, Gray, Haynes \& Richardson, 1996). La principal herramienta que aporta la medicina basada en la evidencia para solventar los problemas mencionados es la implementación masiva del uso de protocolos y guías de actuación. De hecho, diversos autores caracterizan este giro en la medicina a partir de tres elementos que tienen como común denominador el papel central que jugaría el protocolo o la guía. El primero sería el movimiento de la patofisiología a la epidemiología guiada a partir del establecimiento de nuevos protocolos y guías de investigación y actuación. El segundo la creación de protocolos-guías para la propia práctica clínica. Y el tercero, la relación que se establece entre profesionales y legos a partir de las pautas que dictan tales protocolos o guías (Timmermans \& Kolker, 2004). La medicina basada en la evidencia se ha tornado hegemónica en el ámbito de la salud y los datos muestran que en la última década ha habido un incremento exponencial en la elaboración de protocolos y guías. Solamente, por poner un ejemplo bien documentado, en Estados Unidos se estima que se elaboran del orden de un millar protocolos y guías médicas anualmente (Rosser, Davis \& Gilbart, 2001).

Los protocolos y guías ofrecen instrucciones detalladas sobre el proceso de diagnóstico, las pruebas que se deben realizar, cuándo, cómo, y qué intervenciones quirúrgicas se derivan de las anteriores pruebas. También establecen cuánto tiempo deben pasar los pacientes en el hospital, seguir un tratamiento o como retirárselo. La elaboración de un protocolo la realiza un grupo de expertos. Éstos evalúan la literatura científica que existe sobre un problema o circunstancia determinada, la analizan, sintetizan y ofrecen consejos y recomendaciones sobre esa temática atendiendo a la mayor evidencia científica que toda esa información reúne. Los grupos de expertos determinan la audiencia del protocolo o guía, su rango de aplicación, los beneficios que suponen y los problemas o riesgos que implica. No obstante, la construcción de estos protocolos y guías no obedece a un orden lineal, ni mucho menos siempre 
consensuado. Como señalan Knaapen et al. (2010), sus pautas de producción no pueden ser reducidas al intercambio de argumentos y a la búsqueda de acuerdos entre intereses profesionales pre-definidos. La producción de un texto involucra, por un lado, su construcción en sentido literalmente material: sentencias, párrafos, estamentos y formulaciones, que se reajustan y reordenan hasta el cierre obedeciendo a diversos intereses. Y, por otro, diversos actores participan en el surgimiento y estabilización de la configuración del conocimiento y sus prácticas asociadas estableciendo formatos de ensayos clínicos y/o prácticas clínicas.

Los protocolos y guías médicas han recibido cierta atención en las ciencias sociales. En primer lugar se ha examinado el carácter polémico y controvertido de los mismos. Dado que establecen cómo los profesionales deben guiar y realizar su trabajo, entre éstos se han levantado voces que cuestionan la transparencia en su elaboración y la posible participación de terceras entidades (empresas farmacéuticas, agencias políticas, etc.) que implementarían a través de ellos intereses económicos y políticos (Charlton, 1997; Abraham, 1993; Osborne, 1993). En términos más generales, otros autores (Bourret, 2005; Cambrosio et al., 2006) han detallado cómo los protocolos sirven para coordinar dispositivos y mecanismos de diagnóstico e intervención aunque, paradójicamente, ese ejercicio de coordinación antes que eliminar la diversidad de prácticas clínicas y de cuidado entre los profesionales, las transforma y reorganiza. También, en una línea muy similar, se ha examinado con cierto detalle la relación que los protocolos y guías establecen con estructuras de poder. Así, Castel (2009), por ejemplo, ha mostrado que los médicos son capaces de utilizar estratégicamente los protocolos y guías con la finalidad de mejorar su posición en un establecimiento médico y mantener el control sobre las decisiones terapéuticas. En este proceso, no obstante, distintas perspectivas sobre la función de los protocolos-guías y los conocimientos válidos para elaborarlos pueden convertirse en disputas entre especialistas profesionales, académicos y técnicos, cuestionándose en tal dinámica tanto la definición del conocimiento adecuado como los "hechos" que deben formar parte, o no, de su elaboración (May \& Ellis, 2001). Y no es menos cierto, del mismo modo, que otros trabajos han documentado cómo el desarrollo de protocolos y guías contribu- ye a la debilidad del poder profesional y su autonomía, constituyendo, por tanto, una herramienta que es conceptualizada como toda una amenaza por parte del personal sanitario (Timmermans \& Kolker, 2004; Weisz, 2005).

\section{OBJETOS POTENCIALES Y REGÍMENES DE VITALIDAD}

Los primeros protocolos y guías que se elaboraron de manera sistemática hace varias décadas consistían en simples listados con la secuencia de pasos que se debía seguir para establecer un diagnóstico, un procedimiento de atención y un pronóstico. Imitaban abiertamente los protocolos de investigación que se utilizan en laboratorios y centros de análisis clínicos (Berg, 1997). Tal simplicidad ha desaparecido en la actualidad. Los nuevos protocolos han alcanzado tal complejidad que son capaces de construir nuevos objetos, redefinir en sus páginas la propia noción de patología, convertir en problema nuestra normalidad presente y dictar la futura. Para ilustrar esta complejificación retomaremos los tres ejemplos que abren estas páginas.

\subsection{Protocolos en las epidemias}

Como es bien sabido, entre los últimos sucesos epidémicos el estallido de la gripe $\mathrm{H} 1 \mathrm{~N} 1$ ha tenido un impacto social especialmente relevante. Desde su aparición se presentó en los medios de comunicación como una amenaza global (Tirado y Cañada, 2011). Por esta razón, la Organización Mundial de la Salud (OMS; también World Health Organization, WHO) activó unos protocolos cuyo objetivo era actuar sobre toda la población mundial. Desde el inicio del brote, la directora general de esta institución, Margaret Chan, enfatizó en múltiples declaraciones las ventajas de esta situación de "previsión internacional", que, dicho sea de paso, se había venido gestando desde emergencias biológicas previas ${ }^{4}$. Tal entusiasmo se basó en la existencia de unos protocolos que la OMS ha desarrollado con la denominación de "estrategias para toda la sociedad". La novedad de los mismos reside en que esta institución plantea que su actividad debe desbordar sus propios límites institucionales si se desea salvaguardar con eficacia la salud de la población del planeta y no se duda en 
absoluto en entrar en el terreno y competencias de otros actores sociales. Así, "las estrategias para toda la sociedad" afectan e implican tanto a gobiernos nacionales como a organizaciones de la sociedad civil, a comunidades, empresas, familias e individuos. La respuesta que encierran los mencionados documentos de acción está escalada en una serie de dimensiones que van desde el ciudadano de a pie hasta organismos internacionales ${ }^{5}$.

Los nuevos protocolos tienen un amplio espectro de aplicación. Pretenden ser útiles para cualquier emergencia biológica y colocan a la OMS en una posición de total supervisión sobre las agencias de salud estatales y locales. Pero hay mucho más en ellos. Si se examina con atención la Whole-of-Society Pandemic Readiness (WHO, 2009), una guía desarrollada específicamente para el problema de la gripe, descubrimos que sus páginas ponen en relación desplazamientos de personas, estados, agencias de salud, derechos sanitarios, la propia OMS... y diversas dimensiones de nuestra vida cotidiana. De este modo, el protocolo no se limita a establecer recomendaciones sanitarias, también revisa aspectos éticos, relaciones entre instituciones y organizaciones ajenas al sector de la salud, comportamientos individuales y prácticas privadas. La guía, por tanto, es global en extensión, implica a un número incontable de actores, y en intensión, atiende a actividades y comportamientos de todos esos actores. Además, conviene resaltar que los anteriores protocolos vienen acompañados de una serie de mapas e imágenes del planeta que permiten mostrar la evolución de una epidemia en tiempo real, en cualquier lugar en la que esté sucediendo ${ }^{6}$ y establecer la dirección que adquiere el vector de contagio.

Foucault (1976) mostró muy bien que un elemento infeccioso en la época de la medicina clásica se caracterizaba por ser un acontecimiento abrupto, inesperado, que irrumpía en nuestra vida cotidiana sin previo aviso. Se extendía sin regla alguna y el fondo de la epidemia siempre era el momento y el lugar, es decir, la geografía. Así, en el análisis de las epidemias no se imponía reconocer la forma general de la enfermedad, situándola en el espacio abstracto de la nosología, sino bajo los signos generales de un proceso singular variable de acuerdo con las circunstancias de su devenir. La epidemia se singulariza en un espacio y en un tiempo. De hecho, el problema del contagio no era lo más relevante para la medicina. Éste era simplemente una modalidad más del hecho masivo de invasión de la epidemia. A ésta, por tanto, se la debía describir en lo que tiene de singular, de accidental y de inesperada. Se transcribía el acontecimiento al detalle. Y para atender la descripción del fenómeno se designaban médicos que salían del hospital para perseguir a las epidemias y evaluarlas in situ. La tesis foucaultiana sostiene que hay una medicina de las epidemias que se refuer-

\footnotetext{
${ }^{4}$ A título de ejemplo se pueden recordar las siguientes declaraciones: "On the positive side, the world is better prepared for an influenza pandemic than at any time in history. Preparedness measures undertaken because of the threat from H5N1 avian influenza were an investment, and we are now benefitting from this investment". (Chan, 2009)

${ }^{5}$ El siguiente fragmento es un buen ejemplo de lo mencionado:"The purpose and scope of the IHR (2005) are "to prevent, protect against, control and provide a public health response to the international spread of disease in ways that are commensurate with and restricted to public health risks, and which avoid unnecessary interference with international traffic and trade." The IHR (2005) contain a range of innovations, including: (a) a scope not limited to any specific disease or manner of transmission, but covering "illness or medical condition, irrespective of origin or source, that presents or could present significant harm to humans"; (b) State Party obligations to develop certain minimum core public health capacities; (c) obligations on States Parties to notify WHO of events that may constitute a public health emergency of international concern according to defined criteria; (d) provisions authorizing WHO to take into consideration unofficial reports of public health events and to obtain verification from States Parties concerning such events; (e) procedures for the determination by the Director-General of a "public health emergency of international concern" and issuance of corresponding temporary recommendations, after taking into account the views of an Emergency Committee; (f) protection of the human rights of persons and travellers; and (g) the establishment of National IHR Focal Points and WHO IHR Contact Points for urgent communications between States Parties and WHO." (World Health Organization, 2005, p. 1)

${ }_{6}$ "For the first time in history, we can track the evolution of a pandemic in real-time." (Chan, 2009)
} 
za con una policía. Medicina y vigilancia se anudan en esta lógica. Por tanto, Foucault subraya que hablar de epidemias en el siglo XVIII y XIX supone no olvidar la creación de un cuerpo de inspectores sanitarios cuya principal función es perseguir a los agentes patógenos y dar cuenta de su singularidad geográfica.

Sin embargo, los actuales protocolos describen una realidad muy diferente. Ellos muestran un objeto que, en primer lugar, es global, afecta a todo el mundo en todas partes. En segundo lugar, aparece un sentido de homogeneidad permanente. Ese objeto afecta de manera idéntica a y en todo el mundo. $\mathrm{Su}$ identidad se mantiene en Estados Unidos y en España, en Japón y en Austria... por tanto, ese objeto es comparable y equivalente. En tercer lugar, es visible. Se rastrea su pista y movimiento. No existe espacio en el que se oculte. Por último, es un objeto manejable y gestionable. Se visualiza, se sigue y se compara con otros. La conclusión que se extrae inmediatamente de estos hechos es que será cuestión de tiempo que se halle una manera de controlarlo. En definitiva, los actuales protocolos elaborados para enfrentarse al fenómeno de las epidemias producen un objeto infeccioso que es uniforme y comparable en todas sus dimensiones y actuaciones. Se despliega y define en un plano homogéneo y homotético que atraviesa distintas escalas y actores sin sufrir modificación o alteración alguna. En ese proceso, obviamente, sus propiedades permanecen constantes e inalteradas.

\subsection{Protocolos en la teleasistencia}

La segunda de las noticias que abren este texto tiene que ver con el telecuidado y la teleasistencia. Esta modalidad de atención sanitaria se ha tornado muy popular en nuestro país desde que La Ley Española de Promoción de la Autonomía Personal y Atención a las Personas en Situación de Dependencia (Ley 39/2006, 2006), por primera vez en la historia, contempló en su artículo 15 del capítulo II (Prestaciones y Catálogo de servicios de atención del sistema para la Autonomía y Atención a la Dependencia) los servicios de teleasistencia como dispositivos necesarios para promocionar la autonomía personal y la atención a la dependencia. Éstos aparecen definidos con la misma relevancia que otros dispositivos más clásicos como pueden ser los Centros de Día y de Noche, los servicios de Atención Residencial o los Servicios de Ayuda a Domicilio 7.

La teleasistencia o telecuidado se despliega sobre dos grandes ejes. El primero es el uso de las tecnologías de la información y la comunicación y el segundo la implementación masiva de protocolos que pautan absolutamente toda la actividad de la persona atendida y de sus cuidadores. La teleasistencia inaugura un nuevo planteamiento del cuidado. Éste quedará bien ilustrado si nos centramos en un pequeño ejemplo. Nos referimos al denominado SIMAP, dispositivo de teleasistencia móvil. El SIMAP es una iniciativa llevada a cabo por Cruz Roja Española, junto al apoyo privado de la Fundación Alzheimer España. Dirigido específicamente a personas con deterioro cognitivo leve o moderado, este sistema de localización utiliza la telefonía móvil y GPS para determinar la posición de un sujeto con un margen de error de cinco metros. El servicio consta de un pequeño dispositivo móvil con un receptor GPS y un módem GSM que, sujetado a las trabillas del pantalón o falda de las personas afectadas por demencia, permite a la persona autorizada (familiar o cuidador) conocer los movimientos y la localización del usuario en todo momento. Estas coordenadas se actualizan cada tres minutos y, en caso de que el usuario entre en algún edificio o zona sin cobertura, se emite una señal indicando que ésa no es la posición en tiempo real, sino la última recibida. Además, se puede acceder a un historial que guarda sistemáticamente el registro de cada una de las localizaciones detectadas en cada actualización. El acceso a esta información por parte de los cuidadores se realiza a través del Centro de Contacto de la Cruz Roja, de su solicitud vía SMS o por consulta directa en Internet. Así, se permite conocer en todo momento la ubicación de la persona que porta el disposi-

\footnotetext{
${ }^{7}$ En el espíritu, y en la casi literalidad de la ley, existe el ánimo de transformar las antiguas prácticas de cuidado, especialmente las dirigidas a personas con graves déficits físicos funcionales o cognitivos (Tirado, Callén \& Cassián, 2009)
} 
tivo y, gracias a un programa de avisos y alarmas configurados previamente, detectar situaciones potencialmente peligrosas. Para ello, en base a los hábitos y desplazamientos cotidianos de las personas con demencia, los familiares diseñan previamente la cartografía de lo que será el espacio transitable por el usuario. Establecen un espacio geográfico virtual alrededor de la casa del usuario -de 250 a 500 metros aproximadamente-, y son alertados si éste abandona la zona segura. La alarma en la central se activa también en caso de que el aparato detecte que el usuario se desplaza a una velocidad mayor de 35 $\mathrm{km} / \mathrm{h}$, lo que significaría que está utilizando algún medio de transporte.

Los protocolos del SIMAP generan tres efectos interesantes ${ }^{8}$. El primero es la disolución de la frontera entre el espacio social y el espacio de salud y cuidado. El movimiento del paciente se considera parte de su terapia. La vigilancia orgánica y el ejercicio sobre el cuerpo que era típica en el encierro y las intervenciones de restricción deja paso a la acción de la tecnología. Controlando las idas y venidas, considerando los desplazamientos productivos y los improductivos, señalando los espacios considerados sanos e insanos y ofreciendo una respuesta inmediata a cualquier incidente, se está actuando sobre la demencia del paciente. El segundo es la disolución de la barrera entre hogar y vecindario. El territorio de este proyecto es el vecindario. El dispositivo opera precisamente sobre su habilidad de asegurar lo abierto sin recurrir a muros, residencias u hospitales. El vecindario no es exterior ni interior. Es más bien parte de la definición del problema de salud y su recuperación. Esto se observa claramente en un folleto informativo de Cruz Roja en Valencia cuando dice: “¿Salimos a jugar? SIMAP le devuelve su libertad y a ti tu tranquilidad". Y el tercero es la revindicación del movimiento como calidad de vida, tanto de los usuarios como de los familiares y cuidadores. Si el usuario puede salir y el cuidador no está obligado a la vigilancia constante y directa, se reducen las restricciones físicas en el ámbito doméstico, se rebaja la angustia y el estrés del cuidador y aumenta la calidad de vida de todas las personas implicadas en la situación de cuidado. Pero, además, el dispositivo se despliega mucho más allá del plano de la mera solución técnica y ofrece una segunda dimensión de realidad que tiene que ver con el ámbito de la afectividad y el bienestar. En ese sentido, el SIMAP despliega un conjunto de prácticas que implican afectos, reivindicaciones éticas y formulaciones políticas sobre sus usuarios. En ellas, por ejemplo, capital y movimiento se funden en un único valor. El uso del dispositivo supone disfrutar de la movilidad como capital de bienestar tanto para usuarios como para cuidadores. El servicio lo deja muy claro al afirmar qué potencian: "La tranquilidad que proporciona saber que su familiar disfruta de su libertad de forma segura" (Cruz Roja, s.f., p. 5).

$\mathrm{Al}$ igual que ocurría en el caso de los protocolos para actuar en situaciones de emergencia biológica, los protocolos del telecuidado convierten las patologías cognitivas en algo que afecta a diversos actores y cuyo cuidado se despliega en diversas escalas. En ellos, la demencia afecta al paciente, a sus familiares, a los cuidadores, al vecindario, a la comunidad... Y su atención debe movilizar todos estos actores $\mathrm{y}$, de nuevo, transformar comportamientos y prácticas en los mismos. Los protocolos que articulan el telecuidado y la teleasistencia también generan un objeto que se despliega en un plano idéntico y sin variaciones que atraviesa y reorganiza a los mencionados actores y sus conductas.

\subsection{Protocolos en el cáncer de mama}

La palabra cáncer remite inmediatamente a un conocimiento parecido al que expresa la OMS cuando lo define como "un proceso de crecimiento y diseminación incontrolados de células" (OMS, 2010). No obstante, resulta sorprendente descubrir que, por ejemplo, en las actuales guías y protocolos para el cáncer de mama hereditario aparece la siguiente conceptualización:

Su impacto se extiende a los diferentes ámbitos asistenciales y repercute en la actividad, los recursos y la formación de los profesionales de diversas especialidades, a la vez que trasciende

${ }^{8}$ Tirado, Callén \& Cassián (2009).

${ }^{9} \mathrm{El}$ documento se puede consultar en la siguiente dirección:

http://www.cruzroja.es/pls/portal30/docs/PAGE/CCAAVALENCIA/COMUN/SIMAP-CRUZROJA-OK.PDF 
el ámbito sanitario debido a las implicaciones éticas, legales, sociales y económicas que comporta. (Agència d'Avaluació de Tecnologia i Recerca Mèdiques [AATRM], 2006: 21)

Esta guía, por citar un ejemplo, establece que desde un primer encuentro con el especialista se articulan tres escalas de acción típicamente distantes y desconectadas en otro tipo de diagnósticos. En primer lugar, el cuerpo. El protocolo establece la realización de un examen físico detallado que nos lleva inmediatamente a la segunda escala: los marcadores biológicos. Estos son fundamentales en el diagnóstico, siempre deben hacerse presentes y con ellos entramos en la tercera: la familia del paciente. Desde este momento, las tres escalas deben coordinarse para lograr un diagnóstico y estimación apropiada del riesgo de desarrollar la enfermedad. El árbol genealógico es un elemento que, a la vez, enlaza una expansión de la escala familiar: trasciende el tiempo y espacio local, atrae el pasado, lo vuelve presente, $\mathrm{y}$ facilita proyectar el futuro. Mencionar el cáncer en el contexto de la genética oncológica significa necesariamente hablar de muchos cuerpos, reasignando el sentido de esta entidad como una que se encuentra a la base de un cuerpo familiar o de colectivo biológico. Es decir, el cáncer se transforma en un fenómeno extendido (Tirado \& Castillo, 2011). Si bien el diagnóstico se inicia desde el cuerpo del paciente, éste no puede realizarse correctamente sin atender a su examen genético y a la extensión del mismo a los familiares más cercanos. Y sea cual sea la conclusión, el protocolo, basándose y expresando una serie de datos epidemiológicos, establece un estricto seguimiento familiar, el cual implica la realización de pruebas y exámenes periódicos, y medidas de prevención, entre otros aspectos (Tirado \& Castillo, 2011). En todo este proceso actúan también otras escalas del mismo fenómeno del cáncer: una psicológica, que enlaza recursos comunitarios; una ética y legal, que consiste en una serie de enunciados que orientan sobre el valor y justificación de una serie de decisiones, y que se enlaza con una carta de derechos que vuelven a situar la figura del paciente como entidad autónoma. Por lo tanto, en la enfermedad, el cuerpo del paciente es uno de los espacios relevantes, pero no el único: desde un inicio se relaciona con otras escalas que permiten asignarle una u otra cualidad, una u otra posición en la serie de operaciones que compone el algoritmo, un pasado particular y un futuro concreto.

Las guías y protocolos convierten el cáncer en un objeto que es una trayectoria absoluta. Este se convierte en algo que irradia un campo de mediaciones sociales y materiales que se instancia en distintas escalas y espacios. Incluso podríamos señalar que reorganiza circunstancialmente el tiempo, al hacer énfasis en la historia familiar y el genograma como un recurso relevante para estimar el riesgo de poseer la enfermedad. El cuerpo del paciente, el significado de la familia y las relaciones generacionales se resemantizan a partir de un procedimiento técnico y médico. Se ponen en relación de manera diversa y se sitúa otro componente (el cáncer), extraño anteriormente, como eje que articula las relaciones y los afectos. Y, más allá todavía, se puede afirmar que el mismo riesgo se ha convertido en un componente esencial de las intervenciones biomédicas. Si recordamos la noticia citada al comienzo, distintas prácticas, distintos aconteceres, diferentes movimientos, se articulan con una probabilidad (o no) del acontecer de la enfermedad. No obstante, este riesgo no sólo se sitúa en la exterioridad sino que de manera creciente comienza a depositarse en la fuente misma que organiza la vida. Ésta, fundamenta la necesidad de operaciones y activación de rutinas pre-sintomáticas que faciliten o bien la prevención o bien el control. Sea como fuere, el cáncer está ya presente antes de que sea diagnosticado, y los cuerpos, entrelazados por una entidad tan abstracta como un gen ${ }^{10}$ y su posible mutación se reorganizan en torno a la enfermedad, se preparan para su ocurrencia y prevención incluso antes de que hayan aparecido síntomas de su desarrollo.

\footnotetext{
${ }^{10}$ Pese a la variedad de factores o circunstancias carcinogénicas, la teoría predominante a nivel biomédico asume que éstas actúan sólo mediante su efecto a nivel genético. Como señala uno de nuestros oncólogos entrevistados: "La única teoría reconocida y predominante actualmente sobre el origen del cáncer es la genética, no hay otra" (Entrevistado 1, Profesional).
} 


\subsection{Objetos potenciales}

Los tres anteriores objetos, surgidos de un nuevo tipo de protocolos y guías médicas, comparten varias características. Para conceptuar este conjunto de características hemos recurrido a las propuestas de A. N. Whitehead ${ }^{11}$. En ellas existe una interesante diferencia entre la noción de potencia y la de potencial. La primera es un concepto clásico en la filosofía tradicional que hace referencia a una cualidad indeterminada e inespecificada que subyace en los objetos y que adquiere especificación y determinación gracias a la forma. Por tal razón, la tensión potenciaforma constituye un par inseparable y definitorio de toda entidad real. En ese sentido, toda la filosofía clásica ha supuesto que las cosas reales tienen que estar en estado específico o esencial determinado y en estado individual. Tan sólo Dios tenía la cualidad de ser algo real que se hallaba en estado supraindividual o supralocal. Sin embargo, la noción de potencial, que proviene de la física contemporánea, establece para algunos objetos las mencionadas cualidades divinas. Precisamente éstas caracterizan a los campos físicos -gravitatorio, magnético, electromagnético, etc. Tales campos están en todas partes con una eficiencia positiva, se hallan en todos los lugares, en cualquier cuerpo, en las duraciones de todos los fenómenos presentes, pasados y futuros. Mientras que en el caso de la potencia tenemos una cualidad oculta, retirada, que anida en los más profundo de una entidad y, por tanto, espera las condiciones más idóneas que le transfiera la forma para aparecer; el potencial hace referencia a una cualidad siempre presente pero también siempre expresada. La idea de objeto potencial podría resumirse a partir de los siguientes ejes:

1. El objeto potencial no remite a la idea de "potencia" de la ontología clásica, sino a la noción de "potencial" desarrollada por la física moderna (campos magnéticos, electromagnéticos, etc.).

2. Los objetos potenciales ni son abstractos ni ideales, son a todos los efectos actuales y eficientes. Paradójicamente se parecen más a la forma que a la potencia de los antiguos filó- sofos, pero no se limitan ni a la materia ni a la forma.

3. Constituyen una ontología de campo. Su propiedad es la "ubicuidad", estar en todas partes, sin estar contenido, circunscrito, delimitado por ninguna cosa concreta.

4. Esta ontología campal nos lleva a pensar en la posibilidad de la existencia de distintos grados u intensidades ontológicas (Guattari, 1996), pero no diferentes ontologías.

García Bacca (1990:422) establece de la siguiente manera la idea de este tipo de objeto:

La imagen más próxima que un lector no técnico puede formarse de la estructura de un campo es la de imaginar la entidad correspondiente en estado de mar que abarca todo el universo - mar de gravitación (campo gravitatorio), mar de electricidad (campo eléctrico)...- en el cual están como flotando, a la manera de témpanos... las entidades que por otras causas hayan tomado forma más individual, vgr. "este" cuerpo, "este" electrón... Pues bien: los fenómenos reales están regulados por leyes de movimiento -leyes más o menos individuales y sobre individuos definibles físicamente, localizables en tiempo y espacio- y por leyes que expresan la vinculación de tales componentes individuales de la realidad con los componentes supraindividuales o cósmicos, es decir con los campos.

Es decir, son entidades materiales que facilitan y promueven distintos tipos de materialización, sin dejar de ser tan reales o actuales como los objetos o acontecimientos que promueven.

El concepto de Whitehead presenta enormes similitudes con la noción de objeto-mundo propuesta por Michel Serres. Un objeto-mundo suscita una cierta cartografía o geografía del cuasi-objeto, un alcance y una trascendencia de la posibilidad de afectar que va más allá de las configuraciones locales. En palabras de Serres: "Llamamos objeto-mundo a un artefacto en el que al menos una de las dimensiones, tiempo, espacio, velocidad, energía... alcanza la escala del globo" (1991: 32). Tan sólo conociendo la especificidad de la trayectoria que plantea un objeto es posible dictaminar la presencia de un objeto-

${ }^{11}$ Whitehead (1925). Una interesante revisión del trabajo del filósofo ha sido realizada por García Bacca (1990). 
mundo (Mendiola, 2006) y esto también se aplica al objeto potencial. Sin embargo, conviene establecer dos diferencias. En primer lugar, la noción de objeto-mundo no define la posibilidad de la existencia de graduaciones o de distintas intensidades en la medida que se expresa. Refiere la extensión total de una entidad por espacios diversos, pero no permite connotar diferencias en esa explicaciónn. Por el contrario, la idea de potencial es una fuerza que permite establecer estas variaciones y asociarlas a formaciones de entramados diversos que configuran distintas formas de presentación del objeto. En segundo lugar, un objeto-mundo remite a la presencia de una entidad a nivel global. Por el contrario, el objeto potencial se configura, en sí, por relaciones que lo instancian de maneras diversas en distintos espacios y momentos. Su alcance es amplio, trasciende las fronteras que sitúan a los objetos discretos, no obstante, puede o no alcanzar la escala del globo. Cualquiera sea su manifestación, se somete a las escalas y patrones que son producidas mediante diversos dispositivos de regulación. Su propiedad de objeto deviene de, justamente, este aspecto: su cualidad de producto. Mediante distintas estrategias es puesto en relación y se manifiesta a partir de diferentes escalas que enlazan y a la vez se someten a entramados de cualidad socio-material. Y es objeto, pues interactúa, incide y se define por estos intercambios. Por ejemplo, muchos de nosotros, de una u otra manera, nos relacionamos con el cáncer. Este incide en nuestras vidas cotidiana, ya sea para evitarlo o bien para hacerle frente directamente. Ante una crisis biológica - como el caso de la H1N1-, una epidemia, este objeto también pasa a formar parte de nuestro diario vivir. $Y$ en cuanto a la teleasistencia, pese a no situarse en un espacio específico, definible, podemos interactuar con ella, utilizarla, recurrir a sus informes o bien ser sujetos de sus dispositivos de búsqueda y triangulación. Son objetos cuya propiedad principal es la ubicuidad, y su sustancia, la materialidad.
La noción de objeto potencial nos permite entender e integrar en una explicación homogénea todas las nuevas características que presentan los agentes infecciosos de las epidemias, el telecuidado y el cáncer. Estaríamos, por tanto, ante una nueva tipología de objetos, con características campales y homogeneizadoras y productivas, y que se definen a partir del juego de relaciones que establecen unos simples protocolos y guías. Conviene también subrayar que el objeto potencial recoge el espíritu, presente en la teoría del actor-red y buena parte de la microsociología actual que rechaza lo virtual como categoría de análisis social ${ }^{12}$ puesto que hace referencia a una cualidad oculta o perdida en lo material y que espera su momento oportuno para expresarse. El objeto potencial está siempre completamente expresado, nunca es una tensión virtual escondida en alguna materialidad que espera el momento adecuado para actualizarse. Esta nueva tipología de objetos ofrece la posibilidad de hablar de la totalidad como una producción local. Pero una totalidad que desborda los espacios locales manteniéndose constante.

\subsection{Regímenes de vitalidad}

Los objetos potenciales que hemos analizado pertenecen todos al ámbito de la salud y están relacionados con el extremo proceso de protocolarización que sufre la medicina actual. Su aparición tiene para esta disciplina tres consecuencias específicas que merece la pena valorar. La primera y más evidente es que los objetos potenciales redefinen la noción de patología. Como hemos visto en los anteriores ejemplos, los protocolos la deslocalizan del cuerpo y la distribuyen entre innumerables y diversos actores y entidades. En segundo lugar, los objetos potenciales prescriben y reorganizan las relaciones entre todas esas entidades. Establecen cómo es el tipo de relación, su intensidad, su ubicación jerárquica, etc. Por último, esa reorganización supone una reestructuración y un realineamiento de la dicotomía

\footnotetext{
${ }^{12}$ Autores como Bruno Latour (2004) o Sack (1992) han manifestado abiertamente su desconfianza hacia la tensión virtual-actual. En su opinión, lo virtual se define como una propiedad subyacente, oculta y dependiente de otras categorías que detentan la responsabilidad de traerlo a la realidad o no. Esta lógica conduce a una sociología de la sospecha que observa fuerzas ocultas en cualquier indicio. Por el contrario, ellos promueven un pensamiento social que asume que ante nuestros ojos tenemos todos los agentes, materiales e indicios necesarios para hacer un correcto análisis de la realidad social.
} 
patología-normalidad. La enfermedad y la curación se definen y reordenan a partir de la interacción de nuevas escalas y nuevos valores. Cada objeto potencial establece su propio canon para reubicar la tensión salud-patología. A los juegos de relaciones resultantes de cada objeto potencial los hemos denominado "regímenes de vitalidad".

Éstos suponen un re-enfoque de nuestra vida cotidiana, re-activan vínculos, construyen otros nuevos, planifican y desarrollan estrategias para desenvolverse en diferentes niveles de acción e interacción. Constituyen lo que Thévenot (2009) ha denominado una "gramática del vivir-en-común". Pero con una salvedad: los regímenes de vitalidad, en tanto que se fundamentan en un protocolo o guía médica, establecen un juego de verdad. En tales regímenes no se trata únicamente de establecer qué hacer y qué no, qué está bien y qué no lo está; no hay, tampoco, una mera tecnificación de lo viviente, hay, eso sí, un canon que aspira a ser cada vez más verídico sobre qué relación debemos establecer con el acontecer cotidiano que supone la enfermedad y la salud, y, por supuesto, con el conocimiento biomédico. Los regímenes de vitalidad son verdades sobre la vida y cómo vivirla.

\section{REGÍMENES DE VITALIDAD Y MOTILIDAD}

De todo lo dicho anteriormente se desprende que los objetos potenciales suponen una reordenación de la vida. Y si esto es así, tal reordenación debe enfrentarse al interrogante del movimiento, puesto que como ha escrito Serres (1994) existe una estrecha y secreta relación entre movilidad y vida. Para este autor, ésta esconde su secreto en su propagación por el tiempo y el espacio a partir de pequeñas e inesperadas relaciones, de singularidades breves y locales, asociadas mediante cercanías y lejanías. La vida, estrecha y corta, frágil y plegada, obstinada y conectada, debe buscarse en su prolongación singular, en el acontecimiento que siempre abre con su movimiento de expansión ${ }^{13}$. Por tanto, reorganizar la vida supone reorganizar el movimiento. Y los objetos potenciales realizan exactamente esa tarea: crean condiciones para la motilidad ${ }^{14}$. Pero no cualquier condición, ni para cualquier movimiento. Sólo establecen aquella que aseguran un movimiento correcto, saludable o terapéutico.

El movimiento constituye una temática muy joven en la agenda de las ciencias sociales. Paul Virilio y Zigmun Bauman ${ }^{15}$ se cuentan entre los pioneros de su análisis. Ellos han mostrado que el movimiento de personas, cosas, información o ideas, es central en nuestra cotidianidad. Hasta tal punto la temática se ha tornado relevante que John Urry (2007) se ha atrevido a postular que existe todo un "nuevo paradigma de las movilidad" en el pensamiento social. Éste permite visibilizar fenómenos opacos hasta el momento, por ejemplo: a) la aparición de nuevos dispositivos de exclusión que afectan a grandes poblaciones, b) la constitución de redes y movimientos sociales transnacionales, c) la fuerte interdependencia que se establece entre cambios en el movimiento físico y las comunicaciones electrónicas, etc. La movilidad tiene una doble faz. Se presenta, al mismo tiempo, como un análisis de

\footnotetext{
${ }^{13}$ En las páginas en las que Foucault explicita la cuestión del acontecimiento aparece otro tema que también recibe bastante atención: el movimiento o circulación. En tales páginas se habla de circulación de riquezas o monetaria, fuera de las fronteras, urbana, se recuerda la metáfora de la circulación de la sangre en el análisis social, de verdades y personas. Sin embargo, el tema se liquida y cierra de dos maneras harto sencillas: o convirtiendo la movilidad en una función del territorio y del medio, con lo cual la acción importante es la que debe darse sobre este segundo, o transformándola en una dimensión más de la población, con lo que pasa a ser otra variable más que regularán ciertos saberes científicos. En ese sentido, Foucault insiste en que por circulación no hay que entender únicamente la red material que permite la circulación de mercancías o personas, sino la circulación misma, es decir el conjunto de los reglamentos, restricciones, límites o, por el contrario, facilidades y estímulos que permitirán el tránsito.

${ }^{14}$ En nuestra opinión "motilidad" y "movimiento" no son conceptos sinónimos. El primero hace referencia a las condiciones o elementos necesarios que permiten la movilidad. Por tanto, el primero sería algo así como la condición necesaria del segundo.

${ }^{15}$ Las tesis de Paul Virilio sobre el movimiento aparecen sintetizadas en Vitesse et politique. Essai de dromologie (1977), y la llegada de Zigmun Bauman a la temática se da en La globalización. Consecuencias humanas (1999)..
} 
los diferentes sistemas de movimiento que se han desarrollado históricamente y como un argumento que permite analizar nuestro presente. No obstante, como ha señalado Bill Maurer (1999), las recientes discusiones sobre la movilidad se basan en varias asunciones que se manejan de manera completamente acrítica, concretamente, se presupone que el movimiento per se genera cambio, que es autoevidente, y que la característica principal de nuestro presente es la enorme capacidad de movilidad que tenemos a nuestro alcance. Ante este estado de cosas resulta imprescindible preguntarnos qué cuenta actualmente como movimiento y qué cuenta como capital. Mientras que el movimiento de miles de turistas a través de los circuitos internacionales preparados para tal evento es completamente legítimo, la circulación de las últimas familias nómadas de Tuaregs a través de los países que circundan el Sahara se considera ilegítima, está prohibida y perseguida. Frente a la imagen de un movimiento claro, evidente, prístino y etéreo que ofrecen los actuales relatos sobre la movilidad, debemos asumir que nos enfrentamos a una multitud de prácticas que establecen las condiciones de lo que se considerará movimiento o no, movilidad legítima o ilegítima. En suma, la movilidad no es un dato, algo obvio y autoevidente. Constituye un logro precario que exige un esfuerzo y el establecimiento de un conjunto de prácticas y definiciones que lo permiten o lo deniegan. En el espacio de discusión que abre esta afirmación sobre el movimiento y sus condiciones de definición y posibilidad se sitúa nuestra propuesta.

Efectivamente, "régimen de vitalidad" es un concepto que, entre otras cosas, permite analizar y explicar cómo se determinan y se inscriben un conjunto de condiciones de motilidad que permiten cierto movimiento o invalidan otro. Lo hemos observado en el caso de las epidemias. Sus protocolos establecen rutas sanas para que circulen las personas y rutas enfermas que son prohibidas y cerradas. Se genera un movimiento visible para el vector infeccioso y se establecen límites y barreras para la movilidad individual y grupal que circundaría a este vector. Y también aparece con mucha claridad en el caso de la teleasistencia. De hecho, este ejemplo presenta al- gunas peculiaridades sobre las que merece la pena detenerse.

En la teleasistencia, capital y movimiento se funden en un único valor. El uso del dispositivo de telecuidado supone disfrutar de la movilidad como capital de bienestar y afectividad tanto para usuarios como para cuidadores. El servicio SIMAP lo deja muy claro al afirmar -como hemos mencionadoque ellos buscan potenciar: "La tranquilidad que proporciona saber que su familiar disfruta de su libertad de forma segura" (Cruz Roja, s.f., p. 5). A esa movilidad, formulada como capital, que se puede adquirir, comparar o intercambiar, la hemos denominado en otro lugar kinevalor (cinevalor) ${ }^{16}$. Con el término hicimos hincapié en la superposición de una dimensión que hace referencia al movimiento como propiedad física, es decir, como capacidad que detentan los organismos vivos por el mero hecho de ser vida y una dimensión económica. El SIMAP transforma el movimiento en mercancía y, al revés, opera con un objeto mercantil que es la pura motilidad. No obstante, el régimen de vitalidad que se despliega en el telecuidado no reivindica cualquier tipo de movimiento o a éste en un sentido puramente abstracto. Todo lo contrario. Cualquier movimiento no es válido, de hecho, cualquier movimiento no es definido como tal. En el caso de personas con algún tipo de demencia o deterioro cognitivo, sólo el movimiento que permite una localización permanente de esa persona y la detección de situaciones potencialmente peligrosas en relación con su posición geográfica se considera "el buen movimiento". Sin el dispositivo que ofrece tal seguridad, la movilidad es puro riesgo y consecuencia indeseada de un trastorno que se debe controlar. Por tanto, el SIMAP no abre un interrogante por el movimiento como tal. No se trata de buscar el movimiento natural del enfermo o los hábitos de circulación previos al trastorno que esta persona disfrutaba. El dispositivo instala su propia movilidad, con su mapa de circulación geográfica, sus zonas de riesgo, fronteras, etc. El SIMAP ofrece un valor que tiene que ver con una nueva codificación de la potencia perdida de un cuerpo y con el control de la enfermedad o hándicap que se padece. Es decir, establece su propia realidad circulatoria, su

\footnotetext{
${ }^{16}$ Tirado (2009).
} 
propio régimen de motilidad. Y en éste, biología y economía se aúnan en un tejido sin costura.

En el caso del cáncer sucede algo muy parecido. En relación con el cáncer de mama, los protocolos dictan muchas de las condiciones que se consideran ideales para una motilidad que favorezca ya sea el bienestar durante las variantes del tratamiento, la recuperación postoperatoria, o bien que impidan la aparición de complicaciones crónicas. Tales indicaciones suponen la mayoría de las veces modificaciones en la vida cotidiana del paciente y sus familiares y el establecimiento de nuevas prácticas relacionadas con el movimiento. Así lo ilustra el siguiente extracto de un protocolo para cáncer de mama:

En general el ritmo de la vida cotidiana se ve alterado durante los periodos de tratamiento. Durante este tiempo es necesario tener más cuidado de una misma y atender las necesidades que vayan surgiendo, adaptando el ritmo diario a las nuevas situaciones... Cuidarse implica muchos aspectos. La nutrición y mantenerse activa son dos elementos clave para tener más sensación de bienestar... La actividad física también ayuda en este sentido. Hay muchas opciones para hacer actividades, como andar, ir a la piscina, hacer yoga u otros tipos de ejercicio. La actividad aumenta la fortaleza, la energía y también ayuda a disminuir el estrés... Consulte con el equipo asistencial el tipo de actividad que puede desarrollar, éste le asesorará de acuerdo con su situación individual. (AATRM, 2008: 63).

Y también lo exponen algunas de las pacientes entrevistadas en nuestra investigación:

"Está establecido ya como un circuito que cuando hay una intervención de cáncer de mama automáticamente avisan al Departamento de Rehabilitación, porque ya empezaba a informar los ejercicios que tenía que hacer antes de la operación, justo después de la operación; ya presentaba un volante para que después viniera para empezar a hacer el tratamiento del linfedema. Quiero decir, vincular mucho el circuito de rehabilitación con lo que era cirugía y quimioterapia." (Entrevistada 9, Socia gAmis)

Los protocolos sobre el cáncer de mama que hemos analizado combinan condiciones de motilidad concretas con ese nuevo cuerpo producido por las intervenciones biomédicas. Se definen sus nuevas posibilidades, se transforman las antiguas y se dictan las secuencias que deben seguirse y las que se soslayarán sistemáticamente. Así, lo que acontece en la vida cotidiana se cifra en relación con este nuevo valor que adquiere la expectativa biomédica sobre la motilidad de las pacientes. Y no sólo de ellas, sino de todo su entorno social y material.

"Porque realmente [para] cualquier persona diagnosticada la época más dura es desde que te diagnostican hasta que entras en el circuito. Es muy determinado lo que te dicen, mira esto, la mamografía, la biopsia, es un carcinoma, lo que sea, tendrás que operar o empezar el tratamiento; te tienes que hacer analítica, placa, electro, pre-operatorio, tienes que ir a los... empiezas un circuito, pero que este circuito a lo mejor son quince, veinte días o un mes, hasta que te operan." (Entrevistada 9, Socia gAmis)

"Tampoco puedo estar más de dos horas en el ordenador, y eso que... [la fisioterapeuta] ya me enseñó que tengo que estar con el codo apoyado fuerte, porque yo trabajaba asi todo el rato [indica como], pero aún apoyando el codo si estoy las dos horas ya no aguanto, se me hincha la axila y tengo que estar... Y trabajo en eso."

(Entrevistada 2, Socia gAmis)

"Sé que tengo que andar con cuidado con la cocina y taparme, porque cualquier cosa en este brazo... Entonces ando con mucha precaución para que no se forme un linfedema." (Entrevistada 2, Socia gAmis)

En definitiva, los regímenes de vitalidad muestran que no tiene sentido hablar de movimiento en sentido abstracto o al margen de las condiciones que lo definen y posibilitan. En ese sentido, pensamos que el movimiento se fundamenta en unas condiciones de motilidad. Éstas se establecen a partir de prácticas cotidianas muy concretas. Un conjunto de éstas, especialmente relevante en nuestra actualidad, tiene que ver con los regímenes de vitalidad que genera la biomedicina. En ellos, el movimiento adquiere cuatro características que merece la pena recalcar.

En primer lugar, como ya hemos repetido, aparece inscrito en un régimen de relaciones que le otorga 
un sentido concreto. Éste se vincula con cuestiones relacionadas con la vida y con cómo vivirla, con la relación entre salud y patología y con un juego de verdad que sustenta la fundamentación de las mencionadas relaciones. En segundo lugar, y como consecuencia de lo anterior, la movilidad pierde el carácter sociocultural y político que tiene en la mayoría de análisis citados hace un instante y entra de lleno en el terreno de los juegos de verdad y la normatividad científica sobre los que se asientan los regímenes de vitalidad. Con ellos, el movimiento se convierte en buen-movimiento o movimiento-saludable y recomendable. En tercer lugar, también es despojado del carácter fenomenológico que tiene en su relación con el cuerpo. El movimiento expresa salud o enfermedad, es una cura o un intensificador de una patología. En ese sentido, los regímenes de vitalidad sustraen la naturalidad de nuestro cuerpo, la recodifican a partir de una verdad biomédica y nos devuelven unos esquemas de motilidad que se estructuran y organizan a partir de unos modelos o estándares que nada tienen que ver con nuestro cuerpo. Tales modelos se han establecido en un laboratorio y para poblaciones tipo. Por último, el carácter global, total e integral que de manera natural tiene la fenomenología de nuestro movimiento corporal es descompuesto y fragmentado en los mencionados regímenes de vitalidad. Y cuando se nos devuelve convertido en norma biomédica, nos llega parcelado en diferentes secciones que, por un lado, no se articulan entre ellas y, por otro, cada una está regida por un modelo, media o estándar diferente. Es decir, la secuencia de movimientos saludables para los brazos en el caso del cáncer de mama se establece a partir de un canon que se elabora en un laboratorio lejano y que nada tiene que ver con el canon que se elabora para el movimiento de las piernas, tronco o cervicales. Los cuales, a su vez, operan con sus propios estándares, también elaborados en otros centros de investigación especializados en las mencionadas partes del cuerpo.

Lo que muestran tales características no es tanto una denuncia del hecho de que nuestro movimiento dependa de unas condiciones de posibilidad biomé- dicas como que éstas nos devuelven una movilidad fragmentada y en la que hallamos en cada pieza una norma directriz diferente. En suma, los regímenes de vitalidad, a través del movimiento, suponen una captura y reconstrucción de nuestro cuerpo a partir de múltiples estándares biomédicos.

Como hemos señalado, en otro lugar hemos denominado a esta superposición entre movimiento y política cinepolítica (kinepolítica) ${ }^{17}$ y en su actividad el movimiento se torna un valor (cinevalor). No obstante, esta afirmación no es especialmente novedosa. La geografía política muestra que ha habido diversas maneras históricas de enfrentarse a la gestión del movimiento. Sin embargo, la novedad que aportan los regímenes de vitalidad consiste en mostrar la conversión de éste en una de las verdades que deben regir nuestra vida. Si Michel Foucault (1977) mostró cómo el sexo se convertía en sexualidad y pasaba a operar como una de las verdades que el individuo occidental debía utilizar para entenderse, definirse y presentarse ante los demás, ahora, el movimiento está sufriendo un proceso similar. Nuestro presente es el tiempo del buen-cuidado, de la buenasalud, de la buena-naturaleza... y, por supuesto, del buen-movimiento. Y éste es uno de los síntomas más evidentes de que asistimos a toda una reorganización de la vida.

\section{CONCLUSIONES: UNA NUEVA ORGANIZACIÓN DE LA VIDA}

En la última década hay dos autores que han descrito profundas transformaciones en la medicina y han mostrado cómo han supuesto una completa reorganización de nuestra manera de entender la vida y lo vivo. El primero es Alberto Cambrosio ${ }^{18}$. Sus trabajos ilustran muy bien cómo la medicina se ha convertido en biomedicina y la vida se ha articulado a partir de lo que denomina "plataformas biomédicas". En ellas lo vivo es atendido a partir de variables bioquímicas y re-estructurado sobre parámetros biométricos muy precisos y establecidos en los la-

\footnotetext{
${ }^{17}$ Tirado (2009).

${ }^{18}$ Ya hemos citado sus principales trabajos.
} 
boratorios. El segundo es Nikolas Rose ${ }^{19}$. Sus estudios han subrayado cómo las nuevas tecnologías de la información y la comunicación han transformado la medicina de tal manera que ésta se atreve a enfrentarse al desafío de actuar en el nivel de la vida misma. Su mirada se ha tornado completamente molecular y actúa sobre los mecanismos más básicos que hacen de lo vivo algo diferente de lo inánime. En ambos casos tenemos propuestas que ofrecen, en definitiva, descripciones de la re-organización que ha sufrido la vida en las últimas décadas.

Los regímenes de vitalidad apuntan en una dirección similar. Muestran cómo la vida ha experimentado una re-organización reciente en el seno de la biomedicina. Esta vez, sin embargo, a partir de la producción de objetos potenciales que subsisten, en parte, por el desarrollo exacerbado de protocolos y guías de actuación médica. Existirían, no obstante, dos importantes diferencias entre nuestra propuesta y las anteriores. La primera tiene que ver con la atención que prestamos a la materialidad misma de esta re-organización. Sostenemos que algo tan sencillo como un protocolo es el pivoto central de la mencionada rearticulación. Resulta evidente que las grandes transformaciones de los hospitales ${ }^{20} \mathrm{o}$ de los centros de salud son importantes en la práctica médica, la transforman y generan efectos insospechados. Del mismo modo, los avances en el procesamiento y gestión de la información suponen cambios en los procedimientos de diagnóstico, tratamiento e incluso en la comercialización de productos sanitarios ${ }^{21}$. Pero no son menos relevantes la aparición de peque- ños elementos como puede ser una guía o un decálogo de procedimiento. Sus efectos son tan profundos y ampliamente transformadores como los anteriores. En ese sentido no deben descartarse a priori y deben formar parte de nuestro análisis social. La segunda diferencia hace referencia a la diversidad o multiplicidad. Los análisis de Cambrosio y Rose señalan grandes y homogéneas reorganizaciones que afectan por igual a todo el espectro médico. En el caso de los regímenes de vitalidad estaríamos ante una realidad diferente. Cada régimen ofrece su propia reorganización. Los protocolos para enfrentarse a las epidemias, desarrollar e implementar el telecuidado o atender el cáncer de mama conforman cada uno su propio objeto potencial. Éstos tienen características diferenciadas, idiosincrasias propias y ofrecen a partir de sus particulares regímenes de vitalidad organizaciones propias de la vida.

Sin embargo, comparten algo, una dimensión que nos permite hablar en todos los casos de régimen de vitalidad. Como hemos mencionado, todos ellos son juegos de relaciones con cierta estructura u ordenación, ofrecen, por tanto un régimen de actividad. Pero lo más importantes es que en todos los casos existe una voluntad de "optimizar y mejorar la vida". Por esta razón hablamos de vitalidad. Los nuevos protocolos y guías médicas operan sistemáticamente bajo el imperativo de desbordar los límites de lo meramente vivo. Operan bajo el imperativo de empujar la vida hacia sus fronteras para ampliarlos y reconfigurarlos. En ellos, la vida es inseparable de su perpetua re-organización.

${ }^{19}$ También ha sido ya referenciado.

${ }^{20}$ Keating \& Cambrosio (2000).

${ }^{21}$ Rose (2007). 


\section{BIBLIOGRAFÍA}

Aвraham, J. (1993): "Scientific Standards and Institutional Interests: Carcinogenic Risk Assessment of Benoxaprofen" in the UK and US. Social Studies of Science, 23(3) august: 387-444.

Agència d’Avaluació de Tecnologia i Recerca MèdiQues (2006): OncoGuía del consejo y asesoramiento genéticos en el cáncer hereditario. Versión completa. Barcelona: Departament de Salut-Agència d'Avaluació de Tecnologia i Recerca Mèdiques.

Agència d’Avaluació de Tecnologia i Recerca MèdiQues (2008): OncoGuía de mama. Actualización 2008. Barcelona: Departament de Salut - Agència d'Avaluació de Tecnologia i Recerca Mèdiques.

Bauman, Z. (1999): La globalización: consecuencias humanas. Buenos Aires: Fondo de Cultura Económica.

Berg, M. (1997): "Problems and promises of the protocol". Social Science \& Medicine, 44(8): 1081-1088.

Bourret, P. (2005): "BRCA Patients and Clinical Collectives: New Configurations of Action in Cancer Genetics Practices”. Social Studies of Science, 35(1): 41-68.

Bourret, P., Keating, P. \& Cambrosio, A. (2011): "Regulating diagnosis in post-genomic medicine: Realigning clinical judgment?" Social Science \& medicine, 73(6) septiembre: 816-824.

Callon, M. \& Raberharisoa, V. (2008): "The Growing Engagement of Emergent Concerned Groups in Political and Economic Life". Science Technology Human Values, 33(2): 230-261.

Cambrosio, A., Keating, P., Schilich, T., \& Weisz, G. (2006): "Regulatory objectivity and the generation and management of evidence in medicine". Social Science \& Medicine, 63(1): 189- 199.

Castel, P. (2009): "What's Behind a Guideline? Authority, Competition and Collaboration in the French Oncology Sector". Social Studies of Science, 39(5): 743-764.

CHAN, M. (2009): Influenza A (H1N1): World Health Organization [Internet] 29 de abril. Disponible en: http://www.who.int/entity/mediacentre/news/statements/2009/h1n1_20090429/en/index.html [Acceso el 12 de Febrero, 2010).

Charlton, B. (1997): "Restoring the Balance: Evidence-Based Medicine put in its Place". Journal of Evaluation in Clinical Practice, 3(2): 97-98.

CRUz Roja (s.f.): ¿Salimos a jugar? SIMAP le devuelve su libertad y a ti la tranquilidad. Valencia: BancajaCruz Roja.

Cruz Roja probará en Sarria un nuevo sistema de teleasistencia (2011): El Progreso [Internet] 2 de octubre. Disponible en: http://elprogreso.galiciae.com/nova/118538.html [Acceso el 11 de octubre de 2011].

Foucault, M. (1976): El nacimiento de la clínica. Madrid: Siglo XXI.

Foucault, M. (1977): Historia de la sexualidad. Madrid: Siglo XXI.

García BACCA, J. (1990): Nueve grandes filósofo contemporáneos y sus temas. Bergson, Husserl, Unamuno, Heidegger, Scheler, Hartmann, W. James, Ortega y Gasset, Whitehead. Barcelona: Anthropos.

Govt introduces stringent control regulations (2011): Gov introduces stringent control regulations. The Express Tribune [Internet] 7 de octubre. Disponible en: http://tribune.com.pk/story/268568/govt-introduces-stringent-control-regulations/ [Acceso el 11 de octubre de 2011].

Guattari, F. (1996): Caosmosis. Buenos Aires: Manantial.

Keating, P. \& Cambrosio, A. (2000). "Biomedical platforms". Configurations, 8(3) otoño, pp. 337-387.

KeAting, P. \& CAmBrosio, A. (2003): B"eyond "bad news": the diagnosis, prognosis and classification of lymphomas and lymphoma patients in the age of biomedicine" (1945-1995). Medical history, 47(3): 291-313.

Keating, P. \& CAmbrosio, A. (2007): "Cancer clinical trials: the emergence and development of a new style of practice". Bulletin of the History of Medicine, 81(1) primavera:197-223.

Knaapen, L., Cazeneuve, H., Cambrosio, A., Castel, P. \& Fervers, B. (2010): "Pragmatic evidence and textual arrangements: A case study of French clinical cáncer guidelines". Social Science \& Medicine, 71(4): 685-692.

Knoblauch, H. (2005): "Focused ethnography”. Forum: Qualitative Social Research. Sozialforshung, 6(3) septiembre. Disponible en: http://www.qualitative-research.net/index.php/fqs/article/view/20/44 
Landzelius, K. (2006): "Introduction: Patient Organization Movements and New Metamorphoses in patienthood". Social Science \& Medicine, 62(3): 529-537.

Latour, B. (2004): The Pasteurization of France. Cambridge: Harvard University Press.

Ley 39/2006 (2006): Ley 39/2006, de 14 de diciembre, de Promoción de la Autonomía Personal y Atención a las personas en situación de dependencia [Internet]. Disponible en: http://noticias.juridicas.com/ base_datos/Admin/139-2006.html [Acceso el 13 de Marzo de 2007].

Maurer, B. (1999): "Forget Locke?: From Proprietor to Risk-Bearer in New Logics of Finance". Public Culture, 11(2) primavera: 365-385.

MAY, C. \& ElLIS, N. (2001): "When protocols fail: technical evaluation, biomedical knowledge, and the social production of 'facts' about a telemedicine clinic". Social Science \& Medicine, 53(8) octubre: 989-1002.

Mendiola, I. (2006): El jardín biotecnológico. Tecnociencia, transgénicos y biopolítica. Madrid: Los Libros de la Catarata.

Moreira, T., May, C. \& Bond, J. (2009): "Regulatory objectivity in action: Mild cognitive impairment and the collective production of uncertainty". Social Studies of Science, 39(5): 665-690

NANJARI, M. (2011): "Estar sentados durante mucho tiempo puede aumentar el riesgo de cáncer." Las Últimas Noticias, 8 de noviembre, 2011: 10.

Organización Mundial de la Salud (2010): Nota Descriptiva No 297. Cáncer [Internet]. Disponible en: http://www.who.int/mediacentre/factsheets/fs297/es/index.html [Acceso el 16 de Noviembre, 2010].

Osborne, T. (1993): “On liberalism, neo-liberalism and the 'liberal profession' of medicine”. Economy and Society, 22(3): 345-356.

RHEINBERGER, H.J. (2000): "Beyond nature and culture: modes of reasoning in the age of molecular biology and medicine: intersections of inquiry" en: M. Lock \& A. Cambrosio eds. Living and working in the new medical technologies. Cambridge, Reino Unido: Cambridge University Press, pp. 19-30.

Rose, N. (2001): “The Politics of Life Itself”. Theory, Culture \& Society, 18(6):1-30.

Rose, N. (2007): The Politics of Life Itself: Biomedicine, power, and subjectivity in the Twenty-First Century. Princeton: Princeton University Press.

Rosser, W., Davis, D. \& Gilbart, E. (2001): "Guideline Advisory Committee. Assessing guidelines for use in family practice". Journal of Family Practice, 50(11): 969-973.

SACK, H. (1992): Lectures on Conversation. Oxfordsd: Blackwell.

Sackett, D., Rosenberg, W., Gray, J., Haynes, R. \& Richardson, W. (1996): "Evidence-based medicine: What it is and what it isn't'. British Medical Journal, 312(7023): 71-72

Serres, M. (1991): El Contrato Natural. Valencia: Pretextos.

Serres, M. (1994):. Atlas. Madrid: Cátedra.

THÉvenot, L. (2009): “Governing Life by Standards: A view from Engagements”. Social Studies of Science, 39(5): $793-813$.

Timmermans, S. \& Kolker, E. (2004): "Clinical Practice Guidelines and the Reconfiguration of Medical Knowledge". Journal of Health and Social Behavior, 45 Supplement: 177-193.

TiRADO, F. (2009): “Cinepolítica y cinevalor. La "gran transformación” de la biopolítica" en: I. Mendiola ed. Rastros y rostros de la biopolítica. Barcelona: Anthropos, pp. 93-114.

TiRADO, F. y CAÑAdA, J. (2011): "Epidemias: un nuevo objeto sociotécnico". Convergencia. Revista de Ciencias Sociales, 56 mayo-agosto: 133-156.

Tirado, F., \& CASTillo, J. (2011): “Oncoguías-ontoguías: protocolos, panoramas y prehensión en el tratamiento del cáncer". Athenea Digital, 11(1) marzo, pp. 129-153.

Tirado, F., Callén, B. y Cassián, N. (2009): "The Question of Movement in Dwelling: Three Displacements in the Care of dementia". Space and Culture, 12(3): 381-372.

URRY, J. (2007): Mobilities. Cambridge: Polity Press.

VIRILIO, P. (1977): Vitesse et politique. Essai de dromologie. París: Galilée.

Weisz, G. (2005): "From Clinical Counting to Evidence-based Medicine" en G. Jorland \& A. Opinel eds. Body Counts: Medical Quantification in Historical and Sociological Perspectives. Kingston, Canadá: McGill-Queens Press, pp. 377-393. 
Whitehead, A. N. (1925): Process and Reality. New York: Free Press.

World Health Organization (2005): Handbook for Journalists: Influenza Pandemic. Geneva: WHO. Disponible en: http://www.who.int/csr/don/Handbook_influenza_pandemic_dec05.pdf [Acceso el 12 de Febrero, 2010].

Workd Health Organization (2009): Whole-of-Society Pandemic Readiness. Geneva: WHO. Disponible en: http://www.unicef.org/influenzaresources/files/CP045_2009-0808_WOS_Pandemic_Readiness-FINAL.pdf [Acceso el 12 de Febrero, 2010]. 